\title{
¿PARA DEJAR DE HABLAR DE LA TOLERANCIA?
}

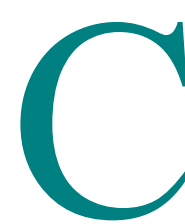

reo que el mejor punto de partida de una reflexión sobre el principio de tolerancia es preguntarse lo obvio: ¿por qué el retorno hoy a la idea de tolerancia? No pocos de los que se planteen esa cuestión convendrán en reconocer que no se trata sin más de una moda académica, y ofrecerán argumentos que vincularán el renovado interés por ese leit-motiv de la Modernidad a los fenómenos de índole social, económica y política que configuran el presente como «horizonte de fragmentación» o «cultura de la diferencia»: el auge de los nacionalismos, la hegemonía del individualismo, el creciente protagonismo de los diferentes movimientos sociales, la preocupación por el estatuto de las minorías, etc.

El debilitamiento de los vínculos que permiten la cohesión e integración en el grupo, otros prefieren hablar de identidad colectiva, como los órdenes normativos (religión, moral social, Derecho), o las instituciones (familia, escuela, mercado de trabajo, Estado), tal y como los había concebido la tradición occidental ${ }^{1}$, parece exigir un especial cuidado en el reconocimiento de lo singular, al tiempo que debilita la posibilidad de llegar a acuerdos que vayan más allá de un marco mínimo.

Es el caldo de cultivo ideal para ese redescubrimiento de la noción de tolerancia al que hoy asistimos. Mi propósito será simplemente mostrar que quienes se ocupan de analizar el concepto de tolerancia ignorando esa dimensión, es decir, perdiendo de vista que se trata de una categoría histórica, yerran no sólo en lo que se refiere al alcance

${ }^{1}$ Me parece que todo ello encuentra uno de sus reflejos más claros en la remodelación de la noción jurídicopolítica de ciudadanía, tal y como ha insistido P. BARCELONA(« I vuoti dell'universalismo giuridico», Democrazia e Diritto, 1989/2, pp. 61 ss) a propósito de la deconstrucción de esa categoría como consecuencia de los fenómenos de racismo y xenofobia. 
descriptivo de su esfuerzo, sino también, y sobre todo, si tratan de proporcionar una dimensión prescriptiva, es decir, cuando concluyen ofreciéndonos la tolerancia como virtud pública a practicar, y aún más, cuando lo formulan como auténtico principio jurídico, incluso fundamental. Adelantaré que lo que trato de hacer ver es algo tan sencillo como que no se puede ignorar la historia, lo que supone tener bien presente, por ejemplo, la ya clásica tesis de MARCUSE/MOORE/WOLFF ${ }^{2}$ y, además, no perder de vista en todo caso que lo que sirvió para abrir el camino hacia el reconocimiento y positivación de derechos y libertades ${ }^{3}$ (de la libertad de conciencia, religiosa, de expresión, etc.) no puede ser hoy proclamado como un objetivo a alcanzar sin retroceder en el estatuto obtenido para las conductas respecto a las que se reclama el «beneficio» de la tolerancia. Habrá que añadir que, en lo que sigue, no me interesa discutir si la tolerancia como «virtud privada» es conveniente y por qué y con qué consecuencias, sino qué significa (y por qué se hace y qué consecuencias tiene hacerlo) proclamar hoy la necesidad de la tolerancia en el orden jurídico político. En otras palabras, no es una propuesta que se sitúe preferentemente en el ámbito de la Filosofía moral, sino en el de la Filosofía jurídica y política, aunque aquí tampoco es fácil ni aun deseable a toda costa la actio finium regundorum...

\section{Sobre el concepto de tolerancia}

Lo primero es tratar de obtener claridad en el concepto: ¿de qué hablamos cuando hablamos de tolerancia? Entre otros ${ }^{4}$, los cuidados

${ }^{2}$ R.P.WOLFF/B.MOORE/ H.MARCUSE, Critique of pure Tolerance, que cito por la trad, alemana, Kritik der reinen Toleranz, Suhrkamp, Frankfurt, 1966.

${ }^{3}$ Es lo que advierte con claridad PECES BARBA, G., Tránsito a la modernidad y derechos fundamentales, Madrid, Mezquita, 1982, que subraya justamente ese carácter de concepto histórico.

${ }^{4}$ Además de los clásicos (desde los precedentes de MARCO AURELIO y SÉNECA y los representantes del Humanismo Renacentista ERASMO, hasta llegar a su formulación en MILTON, LOCKE, BAYLE, DIDEROT o VOLTAIRE, pasando por MONTAIGNE), y del imprescindible trabajo de MARCUSE citado en la nota anterior, habría que referirse ( $\sin$ afán de recoger una bibliografía que es amplísima) por ejemplo a los de G.BUENO, «Sobre la intolerancia», El Basilisco, 4/1978; BOLLINGER, L, The tolerant Society, Oxford/N.York, O.U.P., 1982; PECESBARBA, G., (1982 cit.); VV.AA. (HORTON/MENDUS, eds.), Aspects of Toleration, London, Methuen, 1985; VV.AA., (MENDUS, ed.), Justifying Toleration. Conceptual and Historical perspectives, Cambridge, C.U.P., 1988; VV.AA.,(MENDUS/EDWARDS eds.), On Toleration, Oxford, Clarendon Press, 1988; HOFFE, O. «Pluralismo y tolerancia. Acerca de las condiciones de la modernidad», Estudios sobre teoría del Derecho y de la Justicia, Barcelona, Alfa, 1988; L. FERRAJOLI Diritto e Ragione 
trabajos de A. SCHMIDT, L. GIANFORMAGGIO, J.R. DE PÁRAMO o D. RICHARDS que anteceden, nos proporcionan no pocos elementos de juicio. Sin embargo, creo que en ellos se deja de lado en no poca medida lo que señalaba al principio de estas líneas. Ese concepto «puro» de tolerancia se compadece mal con lo que al fin y al cabo significa, aquí y ahora, adoptar la tolerancia como uno de los criterios de conducta. Veamos.

Las limitaciones de ese análisis de la tolerancia se pueden advertir en el excelente análisis que lleva a cabo GARZÓN VALDÉS. Efectivamente, como explica muy bien GARZÓN VALDÉS, para que podamos hablar de tolerancia, es preciso que concurran tres condiciones: «a) competencia adecuada; b) tendencia a prohibir el acto tolerado... y c) ponderación de los argumentos a favor de la permisión o prohibición del acto en cuestión». El análisis de la situación de competencia adecuada lleva a GARZÓN a distinguir entre «tolerancia vertical» (aquella en la que «se da una relación de supra y subordinación») y «tolerancia horizontal» (en la que se da una situación de igualdad entre los dos sujetos), especies que, según GARZÓN, pueden encontrarse tanto en el ámbito privado como en el público.

El análisis de esos elementos le lleva a añadir otra especificación de la mayor importancia: para que hablemos de tolerancia es preciso que entren en juego dos sistemas normativos, porque el acto o la conducta tolerada presenta una relación intersistemática, una relación de ambivalencia con respecto a dos sistemas normativos: en principio, de acuerdo con el sistema normativo básico, ese comportamiento es objeto de prohibición; sin embargo, de acuerdo con el sistema normativo justificante (es decir, tras la ponderación de buenas razones), se produce la permisión. Pues bien, en mi opinión, los problemas comienzan precisamente por la limitación conceptual de la tolerancia en ese tipo de análisis: aquí se habla de ese concepto preferentemente como propiedad disposicional, y esa es la razón, según creo, de que no sea extrapolable tal concepto de tolerancia al ámbito que más me interesa (en realidad, al que más nos interesa), el público. El primer problema se advierte al analizar la situación de competencia del sujeto que tolera (S1): en efecto, para GARZÓN,

(Teoria Generale del garantismo penale), Bari, Laterza, 1989; F. SAVATER, «La tolerancia, institución pública, virtud privada», Claves de razón práctica 5/1990; PECES-BARBA, Curso de Derechos fundamentales, Madrid, Eudema 1991; GARZÓN VALDÉS, «No pongas tus sucias manos sobre Mozart (Algunas consideraciones sobre el concepto de tolerancia)», Claves de Razón Práctica, no 17/1992; E. FERNÁNDEZ, «Los derechos de las minorías», Sistema 106/1992; V. CAMPS, Virtudes Públicas, Madrid, Espasa, 1991. 
de acuerdo con ALEXY, la tolerancia exige lo que ALEXY llama situación de competencia adecuada (es decir, el status deontico opuesto al de sujeción), esto es, que el sujeto que adopta ese principio se encuentre en una situación de superioridad respecto a aquel otro cuyo comportamiento es objeto del juicio de tolerancia: no hay igualdad. Sin embargo, el mismo GARZÓN modifica esta caracterización, como hemos visto, al admitir junto a la tolerancia vertical la horizontal, la tolerancia entre iguales, en la que «la situación de competencia para prohibir actos entre iguales es expresión de la capacidad que cada cual tiene para defenderse a sí mismo frente a daños y ofensas».

La dificultad nace, a mi entender, de la inadecuación de esa noción de tolerancia más allá del ámbito privado: dicho de otra manera, creo que las situaciones de «tolerancia horizontal» (posibles, sin duda, entre particulares), en el ámbito público son o bien irrelevantes o bien inexistentes ${ }^{5}$, y que la diferencia entre los supuestos de «tolerancia vertical» en el ámbito público y en el privado es tan esencial que hace imposible analogar esas categorías, porque es mucho más importante lo que las diferencia que lo que tienen en común 6 .

Lo que GARZÓN describe como tales («el contrato social hipotéticamente supuesto como remedio a los costos de la anarquía», ola tolerancia recíproca «con miras a crear un cuerpo político civil» en el caso de los cantones suizos 1315 o el acuerdo de los peregrinos del Mayflower) no son ejemplos de tolerancia horizontal, sino expresiones

${ }^{5}$ Me explico: si admitimos (de acuerdo con WARNOCK), que las materias relevantes que pueden ser el objeto de la tolerancia en el ámbito público, son «mandatos de la estética, prejuicios o convenciones sociales», parece claro que nos encontraremos siempre ante una de estas dos situaciones: o bien son tan relevantes que pasan a ser consideradas como bienes jurídicamente protegibles (y en ese momento dejan de ser objeto de tolerancia y se convierten en derechos, lo que constituye el proceso habitual de las convenciones sociales) o bien, por su carácter nimio desde el punto de vista público no se produce reacción, y en todo caso son objeto de tolerancia (privada). Creo que ese es el error de quienes se ocupan de problemas de tolerancia que, en realidad, son problemas de cortesía o educación (ni siquiera especialmente relevantes desde el punto de vista moral) como la tolerancia respecto a hábitos tales como «el desayuno prolongado» o los usos en la indumentaria. El ejemplo del tabaco es suficientemente relevante respecto a cuanto se ha dicho: en el momento en que interviene el Derecho, hoy (ahora, en los países occidentales), ya no cabe hablar de tolerancia respecto a los fumadores: se trata de delimitación de derechos.

${ }^{6}$ En efecto, en el caso de ámbito público, el Sujeto tolerante no está simplemente en una situación de competencia adecuada, sino que por su condición de poder público, dispone del poder normativo. Me parece que esta diferencia, al igual que la anterior, no es captada en el interesante trabajo de P.E. NAVARRO, «Reflexiones acerca del concepto de tolerancia», que he podido consultar antes de su publicación por gentileza del autor. 
de los términos en los que se enmarca el consenso político fundacional, esto es, de derechos: quien pacta en términos de igualdad no pretende tolerancia, sino reconocimiento de garantías y, por tanto, de derechos. En el ámbito público, por definición, como ejemplifican los diversos «Edictos de tolerancia», son objeto de tolerancia comportamientos relevantes pero que no tienen el respaldo de consenso suficiente como para obtener reconocimiento pleno: son otorgados como concesiones de quien está en situación de poder (el rey, el pueblo), es decir, son siempre supuestos de «tolerancia vertical». Por eso, el estatuto que reciba la conducta del sujeto tolerado (S2) está en función de la continuidad de la «buena voluntad» del sujeto tolerante (S1) que ocupa la posición de competencia y frente a la que el sujeto tolerado se encuentra en la de sujeción, y por ello S2 no puede exigir ni reclamar de S1 ser tolerado como un derecho, lo que significa, como advierte RICHARDS, que la tolerancia, en el ámbito público implica siempre un punto de vista paternalista (y casi siempre también en el privado).

En otras palabras, la conducta tolerada es siempre un «mal permitido» ${ }^{7}$, como ejemplifica la clásica denominación de los prostíbulos como «casas de tolerancia» (recuérdese el exabrupto atribuido a CLAUDEL: «itolerancia? Hay casas para eso...»): se trata de un vicio que es mejor soportar para evitar males mayores ${ }^{8}$. Ese argumento prueba la razón del dictum de GOETHE, «tolerar es ofender»: la tolerancia debe ser un régimen provisional, porque en sí encierra siempre algo ofensivo. Por eso me parece especialmente adecuado el análisis de la estructura formal de la tolerancia que propone G. BUENO ${ }^{9}$, y que se basa en la comprensión de la relación circular entre tolerancia e intolerancia, es decir, en la advertencia de que la tolerancia es siempre el resultado de la propia dialéctica de las intolerancias, y no una situación gratuita: la tolerancia incluiría siempre internamente una situación de intolerancia, es una suerte de función respecto a la intolerancia previa (que sería, así, la variable independiente); toda intolerancia implica una tolerancia

${ }^{7} \mathrm{Y}$ así lo enfoca la teología moral propia de la escolástica: la tolerancia es permissio negativa mali; se tolera ad vitanda peiora mala, y cada vez menos en línea con el principio agustiniano Diligite homines, intelficite errores; como lo prueba la Inquisición.

${ }^{8}$ Por esa razón, parece evidente que la tolerancia es la consecuencia de la ponderación de razones justificativas, o al menos prudenciales. En ese caso, la intolerancia, como negación interna de la tolerancia, es algo más que la mera prohibición de un mal: es prohibición que aduce malas razones y por ello resulta más fácil justificar el rechazo en el plano normativo de la intolerancia por carente de justificación que la defensa en el mismo plano de la tolerancia.

${ }^{9}$ BUENO, (1978) cit., especialmente pp. 92-93. 
previa, siendo la tolerancia un proceso intercalado entre dos intolerancias reales o posibles. Esa situación puede simbolizarse así: la situación de intolerancia previa es una influencia de los actos de libertad (Sm1) de S1 sobre los actos de libertad ( $\mathrm{Sm} 2)$ de S2; la reacción de tolerancia de S2 respecto a Sm1 exige «el poder efectivo (causal) o capacidad de causar la interrupción, no ya la influencia de $\mathrm{Sm} 1$ sobre S2 (sobre Sm2), sino la misma constitución de Sm1 (digamos: la producción, acción, emisión por S1 de un contenido m). Si este poder no existe, tampoco cabría hablar de tolerancia, sino simplemente de resignación o paciencia de S2 ante la efectuación por S1 de m». Por eso, puede concluir BUENO el siguiente concepto de tolerancia: «el poder (mejor: el ejercicio del poder) (Sg) de $\mathrm{Sm} 2$ para suspender la capacidad de suspender $(\mathrm{Sm})$ el contenido $\mathrm{m}$ de $\mathrm{S} 1 »^{10}$.

$\mathrm{Y}$ es que, si nos despojamos de la retórica habitual y situamos el principio de tolerancia en el contexto de un Estado de Derecho, en el contexto del reconocimiento de los derechos fundamentales, ese principio pasa a ser algo distinto del tan sufrido «respeto por la diferencia» con el que se le suele identificar.

Más bien, hoy habría que convenir, con FERRAJOLI ${ }^{11}$, que el valor primario de la persona y el principio de tolerancia forman los elementos constitutivos del principio moderno de igualdad jurídica, que, como principio complejo, incluye las diferencias personales y excluye las sociales. Ese «moderno principio de igualdad» es, en mi opinión, y creo que en ello coincidiría con las tesis de PECES-BARBA ${ }^{12}$, corolario de lo que otros significativamente -E. DÍAZ- califican como el núcleo de la «teoría crítica de la justicia», la libertad: «La libertad entendida como eje y fundamento de la legitimidad, lleva al reconocimiento y respeto de las diferencias personales, pero, a la vez, implica como lógica exigencia una básica igualdad -la libertad es la diferencia más la igualdad- y ello tanto en el ámbito de la igualdad en la libertad como en el de la igualdad en la realidad» ${ }^{13}$.

${ }^{10}$ Por eso, para BUENO, la tolerancia así concebida no alcanza la dimensión de concepto moral hasta tanto no pueda ser especificada como buena o mala: dicho de otro modo, cabe hablar de una intolerancia moralmente positiva tanto como de una tolerancia moralmente negativa, según el proceso en cuestión «abra el camino a valores morales positivos o cierre el paso a valores moralmente inaceptables».

${ }^{11}$ FERRAJOLI, (1989) cit., pp.947 y ss. De ahí el nexo biunívoco entre igualdad jurídica y derechos fundamentales. Ello no es óbice para que él mismo insista en la idea de respeto a la diferencia, como constitutivo de un concepto de tolerancia que, según trataré de hacer ver enseguida, por amplio, es hoy en gran medida superfluo.

${ }^{12}$ PECES-BARBA (1991), cit., pp. 245 ss., donde se insiste en la igualdad de trato formal como diferenciación.

${ }^{13}$ Cfr. por ej. las páginas dedicadas a ello en DÍAZ, E., De la Maldad estatal y la 


\section{Tolerancia, ¿para qué?}

Pues bien, si, como es habitual, utilizamos el principio de tolerancia en el sentido amplio en el que lo propone, por ejemplo, FERRAJOLI: «El primado de la persona como valor o del valor de las personas, y por tanto de todas sus específicas y diversas identidades... sobre ese valor se basa la moderna tolerancia, que consiste en el respeto de todas las posibles identidades personales y de todos los relativos puntos de vista... la atribución a cada persona del mismo valor, lo que comporta que intolerancia es el disvalor asociado a una persona como consecuencia de su particular identidad... la tolerancia consiste en el respeto de todas las diferencias que forman las diversas identidades de las personas $\rangle{ }^{14}$ entonces comprobamos que eso que seguimos llamando tole rancia, hoy significa otra cosa, o, si se prefiere, es sustituido con ventaja por otro concepto: se trata, como advierte el mismo autor, de uno de los elementos que entran en la institucionalización moderna de la igualdad jurídica y, en ese caso, ¿qué sentido tiene reivindicar hoy la tolerancia como principio público, y, por consiguiente, exigir su institucionalización jurídica? En mi opinión, y a diferencia de lo que sostienen HÖFFE ${ }^{15}, \mathrm{CAMPS}^{16}$, SAVATER ${ }^{17}$, o Eusebio FERNÁNDEZ ${ }^{18}$ el interés por reclamar la institucionalización de la tolerancia como virtud pública, o, mejor, como principio jurídico y político, desaparece allí donde está garantizada la igualdad y las libertades; en mi opinión, la constitucionalización del pluralismo, la igualdad y las libertades, hace innecesaria la tolerancia en el ámbito público y resuelve las

soberanía popular, Madrid, Debate, 1987 y DÍAZ, E., Ética contra politica. Los intelectuales y el poder, Madrid, CEC, 1990 pp.53 y ss, donde expone su tesis con la que básicamente no cabe estar en desacuerdo del carácter primigenio de la libertad como valor fundante tanto de la legitimidad como de la justicia. La cita corresponde a este último trabajo, p.41. En el mismo sentido, explicitando la existencia de una cláusula general de libertad como clave de bóveda constitucional, PRIETO SANCHIS, L., Estudios sobre Derechos Fundamentales, Madrid, Debate, 1990.

${ }^{14}$ Por eso para FERRAJOLI (1989, pp.947 y ss), valor primario de la persona y principio de tolerancia forman elementos constitutivos del principio moderno de igualdad jurídica, que, como principio complejo, incluye las diferencias personales y excluye las sociales. De ahí el nexo biunívoco entre igualdad jurídica y derechos fundamentales.

${ }^{15}$ HÖFFE, (1988) cit., pp.141 ss.

${ }^{16}$ CAMPS (1990) cit., pp.81 ss.

${ }^{17}$ SAVATER (1990) cit., pp. 30 ss. Sin embargo, como suele suceder en el caso de este filósofo y ensayista, no deja de apuntar, siquiera brevemente (siguiendo el pasaje de GOETHE ya mencionado y reconociendo la razón que asiste, al menos parcialmente, a MARCUSE), el problema al que aludimos, ese carácter transitorio de la tolerancia.

${ }^{18}$ FERNÁNDEZ, 1992, pp.77-78. 
aporías del concepto «puro» de tolerancia, la discusión sobre la imposibilidad del carácter absoluto de la tolerancia ${ }^{19}$. Más aún, allí donde existe ese grado de reconocimiento jurídico, apelar a la tolerancia como principio público es rebajar los derechos (y por ello, entiendo justificadas las tesis de MARCUSE y $\mathrm{WOLFF}^{20}$ ).

El supuesto más claro, hoy, es sin duda el debate en torno a los derechos de las minorías étnicas o, mejor, de los individuos que las componen. Más claramente: el debate en torno a las discriminaciones producidas por el racismo ${ }^{21}$. A ese respecto se aduce, con tanta frecuencia como, en mi opinión, inoportunidad, la idea de tolerancia (no es rara, por ejemplo, la alusión retórica a la convivencia tolerante representada por la Escuela de Toledo en España, cuando se hacen discursos sobre/contra manifestaciones concretas de racismo en o desde nuestro país). Pues bien, ese caso permite advertir sin resquicio de duda lo que trato de hacer ver: reclamar tolerancia para el ciudadano que es diferente en razón de esa diversidad visible -la raza- es emprender un camino atrás en la garantía de sus derechos. Como ha advertido BOBBIO, el problema de la tolerancia de quien es diferente por razones físicas o sociales es un supuesto completamente diferente de la tolerancia de creencias $\mathrm{u}$ opiniones: en el primer caso, se trata de problemas de «prejuicio y discriminación»; en el segundo, el clásico, el problema es «la verdad y la compatibilidad teórica y práctica de verdades contrapuestas $\rangle^{22}$. Es cierto, desde luego, que no se cambia la sociedad por decreto, y que el arraigo de actitudes de respeto y reconocimiento de las creencias, opiniones y diferencias de los otros -un hábito especialmente importante en relación con los «derechos de las minorías»- no es producto automático del Boletín Oficial del Estado, pero no es menos cierto que parece más aconsejable colocar el listón de las exigencias en

${ }^{19}$ Ese es también el error que se advierte en KELSEN, cuando identifica su noción de la justicia con la idea de la tolerancia: ¿Qué es justicia?, Barcelona, Ariel, 1991, p.63.

${ }^{20}$ En el mismo sentido, ROSSET, Lógica de lo peor, Barcelona, Barral, 1975, pp. 192 ss., que concluye: «la tolerancia es palabra de engaño que anuncia, bajo aparente liberalismo, violencias tan intolerantes como aquellas contra las que se subleva».

${ }^{21}$ Sobre ese particular, DE LUCAS, Europa, ¿convivir con la diferencia?, Madrid, Tecnos, 1992 y DE LUCAS, «El reconocimiento de los derechos: ¿un camino de ida y vuelta?», Anuario de derechos y libertades, 1/1992.

${ }^{22}$ BOBBIO, N., ( L'etá dei diritti, del que hay trad. Castellana de F. de Asís, Madrid, Sistema, 1991, por la que cito), p. 244. El problema de la exposición de BOBBIO es, en mi opinión, que no destaca adecuadamente las consecuencias de la dimensión histórica del concepto de tolerancia: precisamente por esa dimensión, hoy carece de sentido plantear los problemas de libertad de conciencia o pensamiento en términos de tolerancia, porque sería un retroceso, como tampoco es admisible enfrentar los problemas de discriminación recurriendo al mismo principio, y no a la igualdad. 
el plano de la garantía del cumplimiento de derechos y no en el de la concesión más o menos graciosa de respeto y reconocimiento de la diversidad. Quizá en todo caso pudiera tener sentido mantener la tolerancia en los términos de la función que históricamente ha desempeñado: el primado del respeto por el otro como diferente constituye históricamente un puente para pasar de la prohibición de determinados comportamientos, reivindicaciones, prácticas o instituciones, a su reconocimiento como derechos, (o en la acepción que presenta $\mathrm{BOBBIO}^{23}$ : la tolerancia como método de persuasión) pero en ningún caso cuando se trata de los que ya han alcanzado ese estatuto formal: aquí la cuestión es garantizar su ejercicio efectivo.

¿Dejar de hablar de tolerancia? Evidentemente, no. La negativa responde, en primer lugar, a la necesidad del recurso a la tolerancia como virtud privada. Pero además, y en el terreno en el que se ha movido este apunte, a su consideración como instrumento provisional, transitorio en el doble sentido del término, en tanto que no sean reconocidos como derechos las conductas en cuestión (y con el fin de facilitar ese tránsito). La tolerancia, hoy, no puede ser un objetivo a reivindicar, sino, en todo caso, un punto de partida desde el que actuar. Es indudable que buena parte de las conductas que hoy se reclaman como derechos (la libertad de opción sexual es uno de los ejemplos más evidentes) comenzaron por aparecer tan sólo como comportamientos respecto a los que parecía posible aducir razones que justificaran su «tolerancia». En mi opinión, hay además un excelente ejemplo de conductas respecto a las cuales sólo es posible (pero también máximamente fundado) el estatuto de tolerancia: me refiero a la desobediencia civil. Entendida en sentido estricto ${ }^{24}$, resulta difícil negar que, si bien no puede recibir el estatuto de derecho (sencillamente porque sería contradictorio que el Derecho reconociera como un derecho la libre facultad de incumplir el ordenamiento jurídico), constituye un bien desde el punto de vista de instrumento para la corrección de la legitimidad democrática. En efecto, mediante la desobediencia civil se trata de apelar a la opinión pública para reconstituir la mayoría en torno a una norma/institución/conducta que se entiende no conforme a las reglas de juego, aunque sea legal: en otras palabras, hay buenas razones que justifican que no se castigue esa conducta, o, al menos, que se responda con el mínimo posible de reprensión ${ }^{25}$. Por eso, estamos ante

${ }^{23}$ BOBBIO, N., (1991) cit., pp. 247 ss.

${ }^{24}$ Cfr. por ejemplo J. MALEM, Concepto y justificación de la desobediencia civil, Barcelona, Ariel.

${ }^{25}$ He tratado de desarrollar este argumento en DE LUCAS, J., «El castigo de los insumisos», Claves de razón práctica, 25/1992. 
un supuesto que encaja perfectamente en lo que hemos descrito hasta aquí como comportamientos «tolerables», sin que ello suponga que pueda dar paso a un derecho ${ }^{26}$.

${ }^{26}$ Salvo, claro está, en el caso de que la presión ejercida por la desobediencia civil consiga que se cambie la norma y se modifique el estatuto jurídico en ella contemplada. Efectivamente, si se trata de un supuesto desobediencia civil directa, podría suceder que una conducta prohibida por la norma objeto de desobediencia civil pasara a ser un derecho. Sin embargo, eso no es más que una de las posibilidades en el amplio abanico casuístico de la desobediencia civil, y, por lo demás, en cuanto tal y por las razones ya aducidas (que se encuentran, entre otros, en KANT), no puede alcanzar el estatuto de derecho. 